\title{
Los valores constitucionales en los jóvenes europeos: singularidades por países
}

\section{Constitutional values in young Europeans: singularities by countries}

\author{
Luis Vicente Amador Muñoz. \\ Universidad Pablo de Olavide. \\ Ivamador@upo.es \\ Francisco Mateos Claros. \\ Universidad de Granada. \\ fmateos@ugr.es \\ Macarena Esteban Ibáñez. \\ Universidad Pablo de Olavide. \\ mestiba@upo.es
}

Francisco Javier Olmedo Ruiz. Universidad de Granada. folmedoruiz@gmail.com

\section{RESUMEN.}

Este trabajo analiza los valores constitucionales europeos de la dignidad, la libertad, la igualdad, la solidaridad, la ciudadanía y la justicia, desde el ámbito de la juventud universitaria de Alemania, Gran Bretaña, España, Francia, Italia y Portugal. En el se reconocen los valores que comparte de manera general toda la juventud europea analizada, y las singularidades de cada país y, además, se aprecia la importancia de una educación en valores ciudadanos, una educación para la ciudadanía. En un primer momento se lleva a cabo un muestreo en el que se buscaron sujetos proviniesen de países con distintos posicionamientos en sus valores y en sus tradiciones democráticas. El instrumento utilizado es un cuestionario de 58 ítems en el que se estudian los valores y las actitudes sociopolíticas en los jóvenes europeos. Posteriormente hemos aplicado la técnica estadística del análisis factorial de correspondencias cuyo objetivo es presentar en un mapa perceptual la información de los datos analizados a partir de tablas de contingencias. En el análisis hemos podido comprobar como las opiniones de los jóvenes difieren en las categorías de apreciación con las que han manifestado su percepción sobre cada uno de los valores estudiados, permitiendo que pueda realizarse un acercamiento definitorio a las características singulares de cada grupo o país a través de los valores constitucionales.

\section{PALABRAS CLAVES.}

Valores constitucionales, juventud, universidad, ciudadano.

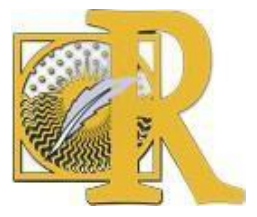




\section{ABSTRACT.}

This paper analyzes the European constitutional values of dignity, freedom, equality, solidarity, citizenship and justice, from the field of university youth in Germany, Great Britain, Spain, France, Italy and Portugal. It recognizes the values shared by all the European youth analyzed in general, and the singularities of each country and, in addition, the importance of education in citizen values, education for citizenship is appreciated. At first, a sampling was conducted in which subjects were sought from countries with different positions in their values and in their democratic traditions. The instrument used is a 58-item questionnaire in which values and sociopolitical attitudes are studied in young Europeans. Later, we applied the statistical technique of the factorial analysis of correspondences whose objective is to present in a perceptual map the information of the analyzed data from contingency tables. In the analysis we have seen how the opinions of young people differ in the categories of appreciation with which they have expressed their perception of each of the values studied, allowing a definitive approach to be made to the unique characteristics of each group or country. Through the constitutional values.

\section{KEYS WORDS.}

Constitutional values, young, university, citizenship.

\section{Introducción.}

Los valores morales y cívicos contenidos en la Declaración Universal de los Derechos Humanos (1948) son comunes a toda la humanidad, su fundamento es la dignidad inviolable de toda persona humana. La UE en la sesión plenaria del 13 de junio de 2003 adopta el texto de la Constitución Europea, que en su artículo dos establece los valores de la Unión basados en los derechos humanos y el respeto a las libertades cívicas, es decir: dignidad, libertad, igualdad, solidaridad, ciudadanía y justicia.

Los valores están muy de actualidad en la medida que son elementos constitutivos de nuestra realidad personal. Las personas regimos nuestras conductas de acuerdo a los valores, incluso antes de descubrirlos o cuestionarlos; así realizamos una serie de acciones, ante situaciones, en las que ponemos en evidencia nuestras preferencias o no, en una palabra, nuestra valoración (Cortina, 2002.). Esta misma autora asigna a los valores la capacidad de estimar objetos, físicos, personales o sociales; nos atraen y complacen; hacen el mundo más habitable; son cualidades que cualifican a las personas, las acciones, las situaciones, las sociedades y las cosas; son positivos o negativos, nos agradan 0 desagradan, nos inducen a actuar en uno u otro sentido. Por ello convenimos en que "es urgente proponer una serie de acciones efectivas, que sean referencias universales como son los valores esenciales referidos a la Declaración de los Derechos Humanos a los que ahora más que nunca, es obligado recurrir" (Pérez Serrano $(1997,11)$

Actualmente llama poderosamente la atención que los jóvenes tienen, en nuestro entorno cultural, comportamientos que no asumen los valores que sustentan los pilares de nuestra sociedad. Una sociedad que basa la convivencia y el disfrute del estado de bienestar en la comunión de una serie de valores que le han permitido participar de una convivencia pacífica y, al mismo tiempo, disponer de acceso a medios y bienes que para otras generaciones fueron impensables. Se abre paso el pensamiento de que esta misma

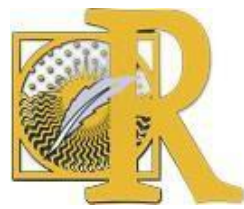


situación genera un estado de desconsideración, por parte de los jóvenes, hacia estos mismos valores. Hace más de dos decenios Grear y Ryan (1989) ya apuntaban que sin valores nuestra sociedad tiene dificultades para continuar y pocas posibilidades de subsistir. Los valores siempre fueron protagonistas de duras batallas en el seno de lo social para dotar a los distintos grupos humanos de derechos y deberes capaces de equilibrar, no sólo la convivencia, sino también las aspiraciones personales en los proyectos de vida individuales. Los valores se erigen, por ello, en orientadores del comportamiento. Ortega y Gasset (1983) dice que el valor preside las decisiones de las personas, puesto que para actuar antes hay que decidir y las decisiones se realizan basadas en las convicciones que se tienen sobre las cosas que nos rodean, sobre las otras personas y sobre nosotros mismos.

Por eso la visión de Durkheim (1975), con respecto al componente cultural de los valores, resulta sumamente interesante por cuanto cada sociedad presenta sus valores y estos varían con relación a los momentos históricos. Rokeach(1979) afirma que los valores se adquieren y se forman durante el proceso de socialización, en su teoría (1968) sitúa los valores sociales en las que él denomina terminales, que son más estables y duraderos, en ellos se reconoce la paz, los derechos humanos, la igualdad y la libertad. Shalon Shwartz (1995) establece dos niveles el individual y el cultural, en este nivel propone unas dimensiones capaces de hacer comprensibles los flujos de cambio en las sociedades: conservadurismo, autonomía, jerarquía, dominio, igualitarismo y armonía. El valor es una orientación individual, es una noción personal de lo deseable, una finalidad que viene a determinar la conducta de las personas. Determinan la compatibilidad o el conflicto que se percibe en la búsqueda y determinación de las metas.

Los valores, por lo especificado, son disposiciones estables de las personas que conllevan preferencias o sentido en las obligaciones (Barton, 1962), determinan preferencias enraizadas en creencias evaluativas de bondad versus maldad o aceptación versus rechazo de un objeto que conlleva la cualidad de obligatoriedad (Fernández García, 2009).

El proceso de socialización proyecta sobre las personas el marco de valores para que ellas adquieran sus preferencias axiológicas (Gimeno, 2009) y en este proceso tienen un papel significativo, además de la familia la escuela, los iguales y los medios de comunicación (Mateos, 1998). No todos tenemos los mismos valores, además de que estos se han ido modificando con el paso del tiempo, acompañando a la propia sociedad. Los valores son también construcción social, son formas y maneras particulares que pertenecen a un determinado grupo cultural (González y Marquínez, 2004), convirtiéndose en lo bueno y valioso de una sociedad y una cultura. Los cambios en la cultura y la sociedad, conllevan y acarrean cambios en los valores del sistema. Nuestra sociedad actual, globalizada, se caracteriza por el constante cambio en los valores y su transformación permanente; lo que viene a subrayar el carácter social y mutable de los mismos.

Esta doble dimensión de los valores: individual y social, constituye niveles paralelos que conforman conductas y respuestas a problemas fundamentales de las personas en su entorno personal y cultural.

La homogeneidad o heterogeneidad de los valores en los jóvenes de distintas sociedades emerge como un indicador de las posibilidades de convergencia entre esas mismas sociedades. Porque si bien convenimos con Cortina $(1995,102)$ que los grandes valores

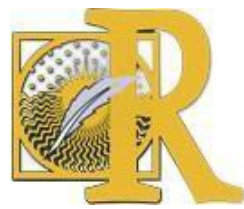


son válidos para todos sin que las épocas o culturas los relativicen, sí que los grupos humanos participando de ellos priorizan unos sobre otros.

Los estudios que se han hecho sobre los valores y las actitudes sociopolíticas en los jóvenes europeos coinciden en señalar que están poco interesados en la política y se muestran escépticos hacia ella (Anduiza, 2001). Esta apatía por la política se recoge también en los jóvenes andaluces (Azcona, 1990). Bernete (1995) encuentra que los jóvenes observan con incertidumbre su futuro, valoran la libertad, la democracia y el conocimiento, así como un cierto orgullo nacional. En Andalucía (Consejería de Asuntos sociales, 1996) los jóvenes no se diferencian de los europeos, y en la percepción de los valores democráticos (Pérez Serrano, 2003) tampoco se distinguen del resto de los jóvenes europeos.

Hay similitudes en valores sociales, pero Orizo (1991) encuentra que los españoles valoran más la familia, la religión y el trabajo. Pérez Serrano (1998) halla similitudes entre los jóvenes españoles, los alemanes, los irlandeses y los estadounidenses en madurez, igualitarismo y actitudes democráticas. En general, los jóvenes se perciben tolerantes, valoran las actitudes democráticas y tienen una concepción del buen ciudadano como no participante en la política y sí cumplidor con sus deberes como ciudadano (Pérez Serrano, 2000). Tienen alta estimación sobre la tolerancia, la democracia, la igualdad, la justicia y carecen de conocimiento sobre las instituciones públicas (Solís, 2005) y, aunque aprecian la participación social se inclinan por las organizaciones y las redes informales, manifestando un rechazo por las organizaciones sociopolíticas (Vals Iparraguirre, 2005) y un recelo hacia los sindicatos (Antón, 2007; Monreal, Mateos et al., 2012).

Los valores no son solamente objetivos o subjetivos, son ambos conjuntamente. Es el sujeto el que valora lo que el objeto ofrece para ser valorado; todo lo que favorece la realización plena de la persona podemos reconocerla como un valor. Todos estos valores se han implantado en las normativas europeas y en sus principios de vida en común, convirtiéndose en baluarte de las políticas sociales

En nuestro estudio, desarrollado en el marco del proyecto I+D: "Los valores constitucionales y las actitudes democráticas de los jóvenes europeos" (2005/2009), lo movía el interés por saber cómo perciben los valores sobre los que se apoya la Constitución europea. Si se manifiestan estas coincidencias y cuáles son las prioridades que se establecen en estos valores sociales entre los jóvenes de estos países estudiados.

\section{Metodología.}

El estudio se realizó con una muestra de 1037 jóvenes de los que el 72,1\% son mujeres y el $27,9 \%$ son hombres de seis países de la Unión Europea, repartidos en un 33,5\% de España, 17,2\% de Italia, 5,9\% de Gran Bretaña, 12,4\% de Francia y 12,2\% de Alemania. La mayor parte de ellos (78,2\%) son estudiantes, un $10,1 \%$ trabajan y el $11,8 \%$ restante ni estudia ni trabaja. En cuanto al nivel de estudios el 0,8\% tiene estudios primarios, el 39,7\% superó los estudios de secundaria, el 6,4\% estudios de formación profesional específica, el $35,9 \%$ posee una diplomatura universitaria y el 17,2\% alcanzó el nivel de licenciatura. Civilmente el $90,4 \%$ están solteros, el $4,2 \%$ son casados y el 5,4\% restante se encuentra en otro estado civil $(0,1 \%$ divorciados, etc.). En el muestreo se ha buscado especialmente que 
los sujetos proviniesen de países con distintos posicionamientos en sus valores y en sus tradiciones democráticas.

El instrumento utilizado es un cuestionario, muy amplio para cubrir los objetivos de la investigación $(\mathrm{l}+\mathrm{D}+\mathrm{l})$ fue readaptado de otro ya utilizado en investigaciones previas (Pérez Serrano, 2003) y actualizado en una investigación realizada en marzo de 2017.La subescala de valores sociales elaborada para esta ocasión consta de 58 ítems. El instrumento arroja un coeficiente de fiabilidad (alfa de Cronbach) de .78 que comparado con el .85 de las otras investigaciones resulta adecuado. Considerando que ya ha sido utilizado en tres ocasiones con validez constatada, hemos procedido a realizar un análisis factorial para analizar la consistencia interna con una parte de la muestra (España, Italia, Portugal, Reino Unido y Alemania) y compararlo con el mismo análisis de la muestra completa. Los resultados de este procedimiento de validez cruzada demostraron similitud entre las variables que saturan cada factor confirmando su validez.

Para establecer el perfil por países de los valores constitucionales seleccionamos los ítems correspondientes a cada valor, transformándolos en una única variable que lleva el nombre del valor. Posteriormente hemos aplicado la técnica estadística del análisis factorial de correspondencias cuyo objetivo es presentar en un mapa perceptual la información de los datos analizados a partir de tablas de contingencias (Díaz, 2002). Dar una visión de proximidad o lejanía entre las categorías de las variables (Etxeberría et al., 1995), en nuestro caso valores constitucionales y países y establecer cuáles son los valores constitucionales que pueden significar a los jóvenes de la muestra en cada país.

\section{Resultados.}

\subsection{Dignidad humana.}

El art. $1^{\circ}$ de la Declaración Universal de los Derechos Humanos se dice: "todos los seres humanos nacen libres e iguales en dignidad y derechos y dotados como están de razón y conciencia, deben comportarse fraternalmente los unos con los otros". La persona y su dignidad son los fundamentos de los valores, de manera que los marcos socioconvivenciales de los que se dotan los países se hacen a la luz del valor de la dignidad humana, o lo que es lo mismo, este valor es el eje principal del fundamento ético de todo sistema democrático. En nuestro estudio, la mayoría de los jóvenes $(92,9 \%)$ coinciden en que todas las personas son merecedoras de respeto y protección. Aplicando el análisis factorial de correspondencias se observa que hay matices diferenciadores en esa apreciación.

Tabla 1. Resumen.

\begin{tabular}{|c|c|c|c|c|c|c|c|c|}
\hline \multirow[t]{2}{*}{ Dimensión } & \multirow[t]{2}{*}{$\begin{array}{l}\text { Valor } \\
\text { propio }\end{array}$} & \multirow[t]{2}{*}{ Inercia } & \multirow[t]{2}{*}{$\begin{array}{c}\text { Chi- } \\
\text { cuadrado }\end{array}$} & \multirow[t]{2}{*}{ Sig. } & \multicolumn{2}{|c|}{ Proporción de inercia } & \multicolumn{2}{|c|}{$\begin{array}{c}\text { Confianza para el Valor } \\
\text { propio }\end{array}$} \\
\hline & & & & & Explicada & Acumulada & $\begin{array}{l}\text { Desviación } \\
\text { típica }\end{array}$ & \begin{tabular}{|c|} 
Correlación \\
2
\end{tabular} \\
\hline $\begin{array}{l}1 \\
2 \\
3 \\
\text { Total }\end{array}$ & $\begin{array}{r}, 289 \\
, 145 \\
, 084\end{array}$ & $\begin{array}{l}083 \\
, 021 \\
, 007 \\
111\end{array}$ & 114,187 & $.000^{\mathrm{a}}$ & $\begin{array}{r}, 749 \\
, 188 \\
, 064 \\
1,000\end{array}$ & $\begin{array}{r}, 749 \\
, 936 \\
1,000 \\
1,000\end{array}$ & $\begin{array}{l}, 034 \\
, 030\end{array}$ &, 034 \\
\hline
\end{tabular}

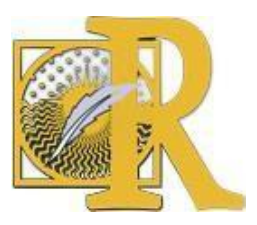


En el análisis, las dos primeras dimensiones (tabla 1) definen el 99,7\% de la inercia que vienen referidas, en la primera a las categorías de respuestas positivas con respecto a la percepción del valor (algo de acuerdo y totalmente de acuerdo), y en la segunda a las respuestas negativas (algo en desacuerdo y totalmente en desacuerdo). Italia y España son los países que contribuyen en mayor medida a la inercia en la primera dimensión (tabla2).

Tabla 2. Examen de los puntos de fila.

\begin{tabular}{|c|c|c|c|c|c|c|c|c|c|}
\hline \multirow[t]{3}{*}{ País } & \multirow[t]{3}{*}{ Masa } & \multicolumn{2}{|c|}{$\begin{array}{c}\text { Puntuación } \\
\text { en la } \\
\text { dimensión }\end{array}$} & Inercia & \multicolumn{5}{|c|}{ Contribución } \\
\hline & & \multirow[t]{2}{*}{1} & \multirow[t]{2}{*}{2} & & \multicolumn{2}{|c|}{$\begin{array}{l}\text { De los puntos a } \\
\text { la inercia de la } \\
\text { dimensión }\end{array}$} & \multicolumn{3}{|c|}{$\begin{array}{l}\text { De la dimensión a la } \\
\text { inercia del punto }\end{array}$} \\
\hline & & & & & 1 & 2 & 1 & 2 & Total \\
\hline España & ,338 & ,476 & ,382 & ,029 & ,265 & ,341 & ,756 & ,244 & 1,000 \\
\hline Italia & 173 & $-1,090$ & 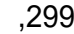 & ,062 & ,714 & , 107 & ,963 & ,036 & 1,000 \\
\hline Portugal & ,050 & ,273 &,- 265 & ,003 & ,013 & ,024 & ,358 & 170 &, 528 \\
\hline Gran Bretaña & ,126 &, 134 &,- 133 &, 001 & ,008 & ,015 & ,639 & ,317 & ,956 \\
\hline Francia & ,191 &,- 003 &,- 531 & ,009 &, 000 & ,372 & ,000 & ,866 & ,866 \\
\hline Alemania & ,122 &,- 013 &,- 407 & ,007 & ,000 &, 140 & ,001 & ,400 & ,401 \\
\hline Total activo & 1,000 & & & 111 & 1,000 & 1,000 & & & \\
\hline
\end{tabular}

Sin embargo, en el análisis gráfico (gráfico.1) se puede apreciar que España tiene alta presencia en la categoría "totalmente de acuerdo" (extremo positivo de la primera dimensión); mientras Italia se manifiesta en la categoría "algo de acuerdo (extremo negativo de la primera dimensión); Francia lo hace en las categorías de desacuerdo y Alemania, Portugal y Gran Bretaña se establecen con una presencia más moderada en las categorías de acuerdo, es decir en apreciación con el valor de la dignidad humana Como las desviaciones típicas son pequeñas (Tabla 1) los resultados serían muy similares con otras muestras de igual tamaño, pertenecientes a la misma población y con el mismo procedimiento (Díaz, 2002). En consecuencia, se puede afirmar que los jóvenes españoles aprecian más que el resto de los jóvenes de los otros países estudiados el valor de la dignidad humana, en el lado opuesto estarían Francia y Alemania.
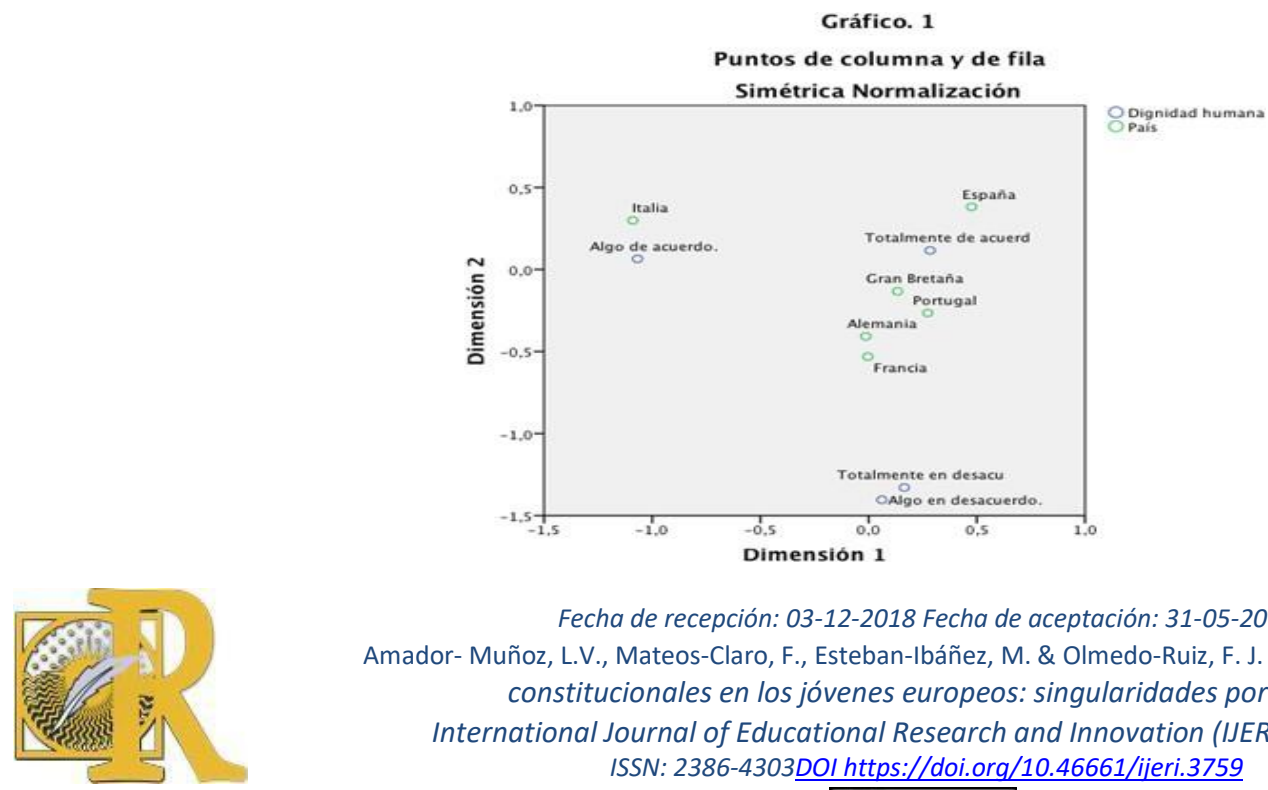


\subsection{La vida.}

La vida es el valor fundamental, sin él no tienen sentido los demás valores. El valor de la vida es la aceptación del derecho de toda persona a existir y a disfrutar de sus facultades para comunicar y relacionarse con el resto de miembros de la sociedad. La vida como valor se sustenta en el estudio mediante dos cuestiones que aluden al valor de la vida en el país y la aplicación de la pena de muerte (Monreal, Mateos et alter, 2012, 50). La mayor parte de los grupos de jóvenes estudiados perciben el valor de la vida $(68,8 \%)$ y apoyan un espacio de convivencia libre de la pena de muerte. Al igual que en valor anterior queremos conocer cuál la percepción que tienen por países, también en este caso son dos las dimensiones que se aplican al diseño del análisis, con ellas se explica el 93,6\% de la contribución a la inercia de las dimensiones. Las categorías de "totalmente en desacuerdo" que se interpreta como percepción positiva con respecto al valor de la vida, y "algo de acuerdo" que es una percepción algo negativa del mencionado valor, conforman la primera dimensión que contribuye con un $57,7 \%$. La segunda dimensión con una contribución del $35,9 \%$ se encuentra saturada por las categorías de "totalmente de acuerdo" y "algo en desacuerdo" en las que se repite la valoración negativa y positiva respectivamente.

Italia $(, 509)$, Gran Bretaña $(, 265)$ y Portugal $(, 148)$ son los países que contribuyen en mayor medida a la inercia de la primera dimensión, y Alemania $(, 740)$ destaca en su contribución a la inercia en la segunda dimensión. Para interpretar más precisamente la percepción del valor acudimos al análisis gráfico (gráfico 2) en él observamos que Italia está posicionada con alta presencia en la categoría "algo de acuerdo" (extremo positivo de la primera dimensión), situación que comparte también Portugal. Gran Bretaña, por su parte, ocupa la posición cercana a la categoría "totalmente en desacuerdo" (extremo negativo de la primera dimensión).

Alemania tiene alta presencia en la categoría "totalmente de acuerdo" (extremo negativo de la primera dimensión), siendo el país que menos valor manifiesta por la vida. Italia y Portugal estiman en menor medida que Gran Bretaña, Francia y España este valor por la vida.
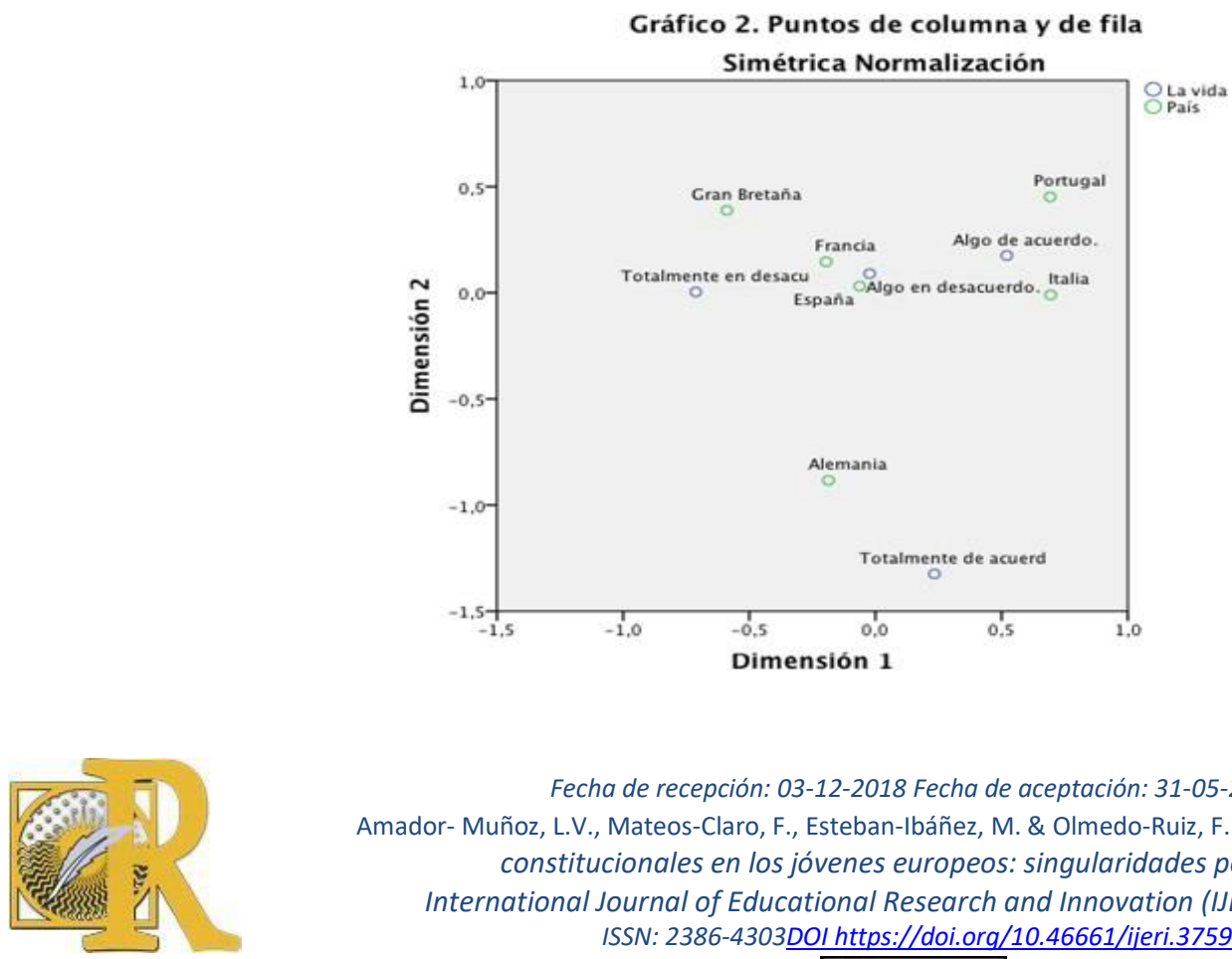
3.3. La integridad de las personas. El valor de la integridad se refiere a la fortaleza de las convicciones que hace que las personas permanezcan fieles a sus principios en todos sus comportamientos, haciéndolo extensivo a todas las situaciones en las que participan o se ven inmersas. Se manifiesta en el derecho que tienen las personas a mantener la integridad física, psíquica y moral. En el caso de la integridad física se refiere a preservar íntegramente el cuerpo y su estado de salud, la integridad psíquica significa preservar las habilidades intelectuales, emocionales y motrices, y la moral a desarrollar la vida de acuerdo con las convicciones personales. En el estudio se han utilizado cinco cuestiones para analizar el valor de la integridad, que se refieren al consentimiento informado, el comercio con el cuerpo, la tortura y la obligatoriedad de trabajar en el caso de los inmigrantes y de aquellas personas que no quieren hacerlo. Los grupos de jóvenes estudiados se manifiestan más a favor del valor de la integridad de las personas $(51,5 \%)$, pero, en este caso, hay un elevado porcentaje de aquellos que perciben más negativamente el valor de la integridad.

El análisis en profundidad permite concretar este aspecto en los distintos países. Dos dimensiones explican el $99,7 \%$ de la inercia, aunque ésta se acumula prácticamente en la primera con el $89,6 \%$, que contiene las categorías de "algo en desacuerdo" y algo de acuerdo".

Examinando la contribución por países Italia $(, 440)$, España $(, 239)$ y Francia $(, 157)$ tienen mayor carga en la primera dimensión, Alemania $(, 587)$ y Gran Bretaña $(, 205)$ lo hacen en la segunda. Recurriendo al análisis gráfico (gráfico 3), España y Francia se asocian a la categoría "algo en desacuerdo" e Italia y Gran Bretaña con "algo de acuerdo" mientras que Alemania lo hace con "totalmente de acuerdo". De acuerdo con estos resultados los españoles y los franceses valoran más la integridad de las personas que los italianos y los británicos; con una menor valoración se sitúan los alemanes.
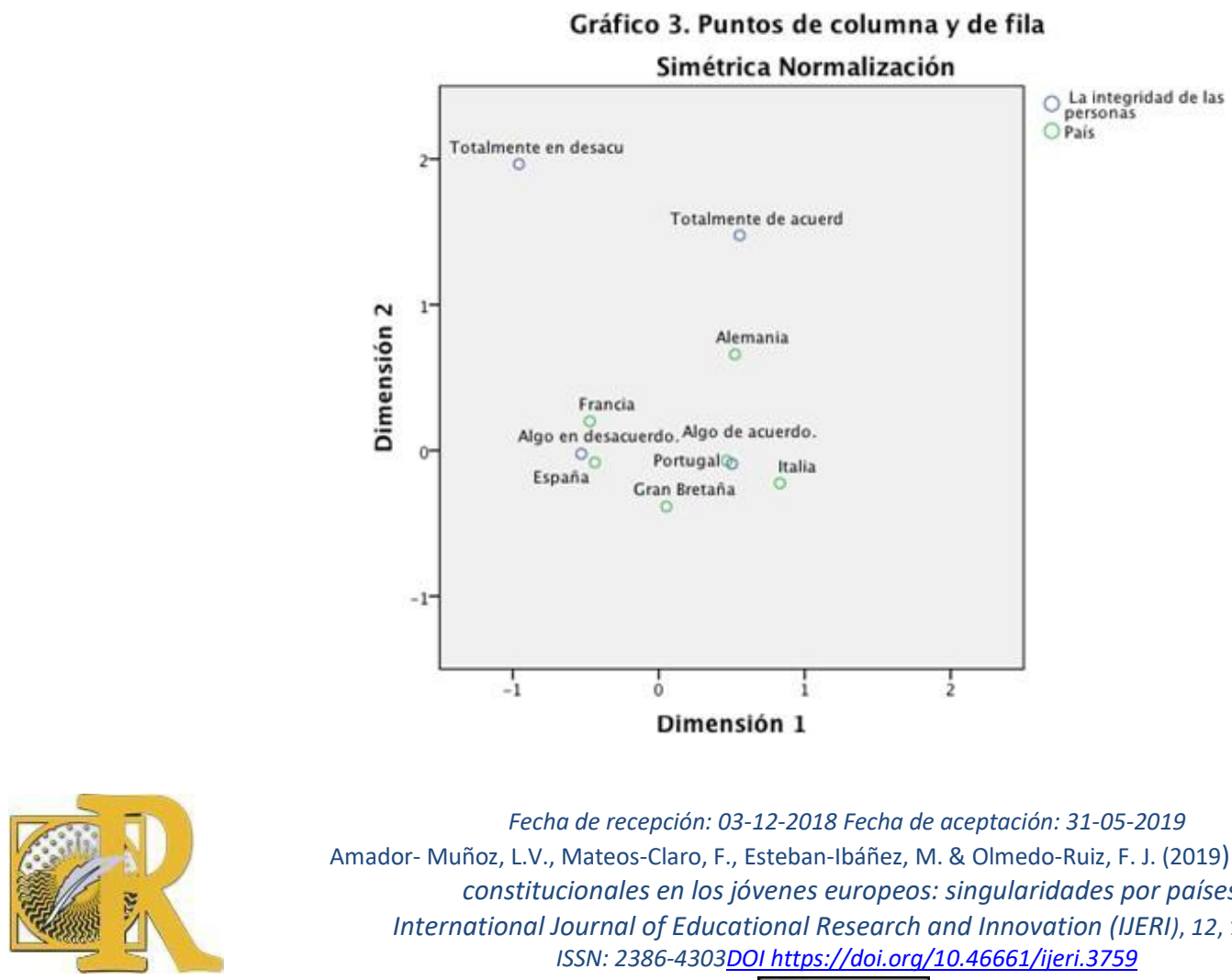


\subsection{La libertad.}

Se entiende la libertad como capacidad para decidir por sí mismo, la autodeterminación y el gobierno propio, y en el terreno social el derecho a participar en las decisiones colectivas. Para Thiebaut $(1999,72)$, "La libertad es ideal y principio ético, político y jurídico que se refiere a la construcción de las acciones de los individuos y a la capacidad que tienen como sujetos para desarrollar sus ideas, planes de vida o propuestas". Integran este valor doce cuestiones (opus cit. 51) que se refieren a la concepción de las libertades: individual, de expresión, política, al cumplimiento de las normas por parte de los extranjeros, la necesidad de tener principios, al respeto a la vida privada y familiar y a las libertades de acceso a la educación y al mercado laboral. El 96,9\% de los grupos de jóvenes están de acuerdo con el valor de la libertad.

Analizamos más en concreto este aspecto para determinar las características por países. Dos dimensiones explican el $97,7 \%$ de la inercia, correspondiendo el $71,4 \%$ a la primera, que se corresponde con las categorías positivas, y el $26,3 \%$ a la segunda con las categorías negativas o de desacuerdo.

Las contribuciones por países nos definen a casi todos ellos aglutinados en torno a la primera dimensión, Italia $(, 435)$, Alemania $(, 274$ y ,396 en la segunda) presentan un mapa en el que la concentración se reafirma en que todos valoran la libertad. El gráfico (gráfico 4) nos presenta esta situación, aunque en él puede apreciarse como Portugal se asocia a la categoría totalmente de acuerdo (extremo positivo de la dimensión) destacándose algo del resto de países, mientras que Alemania y Gran Bretaña lo hacen suavemente hacia la categoría de "algo en desacuerdo". Lo que nos permite inferir que son los portugueses los que destacan en valorar la libertad y los que menos, sin destacar los británicos y los alemanes (gráfica 4).

Gráfico 4. Puntos de columna y de fila

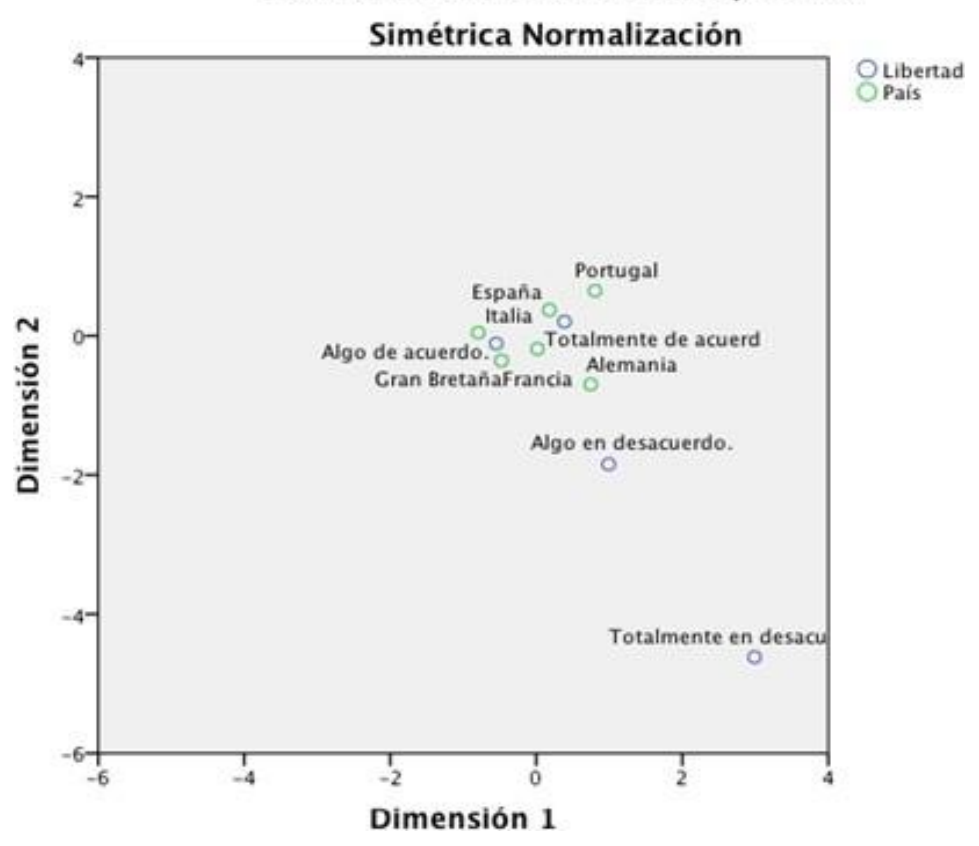




\subsection{Igualdad.}

El artículo 14 de la Constitución Española: "Los españoles son iguales ante la ley, sin que pueda prevalecer discriminación alguna por razón de nacimiento, raza, sexo, religión, opinión o cualquiera otra condición o circunstancia personal o social." Define perfectamente el valor de la igualdad y cómo debe ser interpretado en el seno de la sociedad. En el estudio el valor de la igualdad está contenido en cuatro cuestiones que se refieren a la igualdad de oportunidades para todos, que el sistema de igualdad sea efectivo y la promoción de medidas de igualdad para los sectores desfavorecidos.

El $94,2 \%$ de los jóvenes estudiados están de acuerdo con este valor, pero nos interesa saber cuáles son las matizaciones por países. En el análisis de correspondencias dos dimensiones explican el $95,4 \%$ de la inercia. La primera dimensión $(60,7 \%)$ con la categoría "totalmente de acuerdo" y la segunda (34,3\%) para las categorías de desacuerdo. El nivel de coincidencia entre los distintos países, en la igualdad se aprecia en las contribuciones, apenas ninguna destacada.

El gráfico 5 confirma esta apreciación, aunque resulta más esclarecedor. En él se aprecia como en el extremo positivo de la primera dimensión se sitúan España y Gran Bretaña que se asocian con la categoría "totalmente de acuerdo", Italia se asocia con la categoría "algo de acuerdo", en el extremo negativo de la misma dimensión. En este caso, teniendo en cuenta la concentración, tal y como se ve en el gráfico citado, puede decirse que prácticamente todos los países son coincidentes en su asociación con las categorías, singularmente las positivas, de acuerdo, sin que se pueda perfilar a alguno con respecto a los demás.

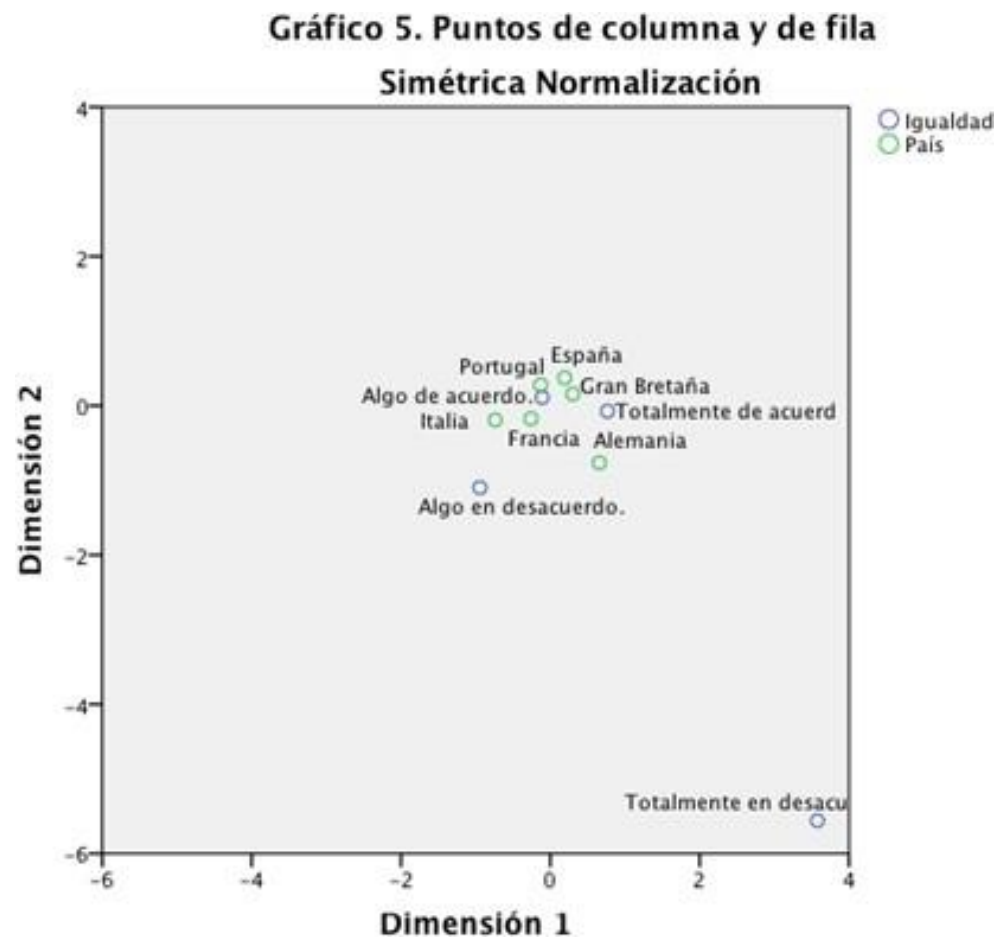




\subsection{Solidaridad.}

El valor de la solidaridad está sustentado por el valor de la igualdad y se manifiesta en el reconocimiento del bien común para todas las personas y responder favorablemente ante las necesidades de los demás. En el estudio se corresponde con once cuestiones den relación con la marginación, la colaboración entre todos, el derecho a la protección social, a la sanidad, a la familia, a los desfavorecidos, al medio ambiente y a la ayuda en la situación de paro laboral.

En el estudio el 95,9\% de los grupos de jóvenes están de acuerdo con este valor. El análisis factorial de correspondencias revela dos dimensiones en la que el mayor peso se encuentra en la primera $(94,8 \%)$ determinada por las categorías "algo de acuerdo" y "totalmente de acuerdo". Italia $(, 548)$, Francia $(, 170)$ y Gran Bretaña $(, 169)$ son los países que más contribuyen en la dimensión. Observando el gráfico (gráfico 6) se aprecia que Gran Bretaña (extremo positivo de la dimensión) y Francia (extremo positivo) se asocia a la categoría "totalmente de acuerdo", que Italia (en el extremo negativo de las dos dimensiones) se asocia a la categoría "algo de acuerdo". La percepción de solidaridad es más favorable en los grupos de jóvenes británicos y franceses, y menos en los grupos italianos.

\section{Gráfico 6. Puntos de columna y de fila}

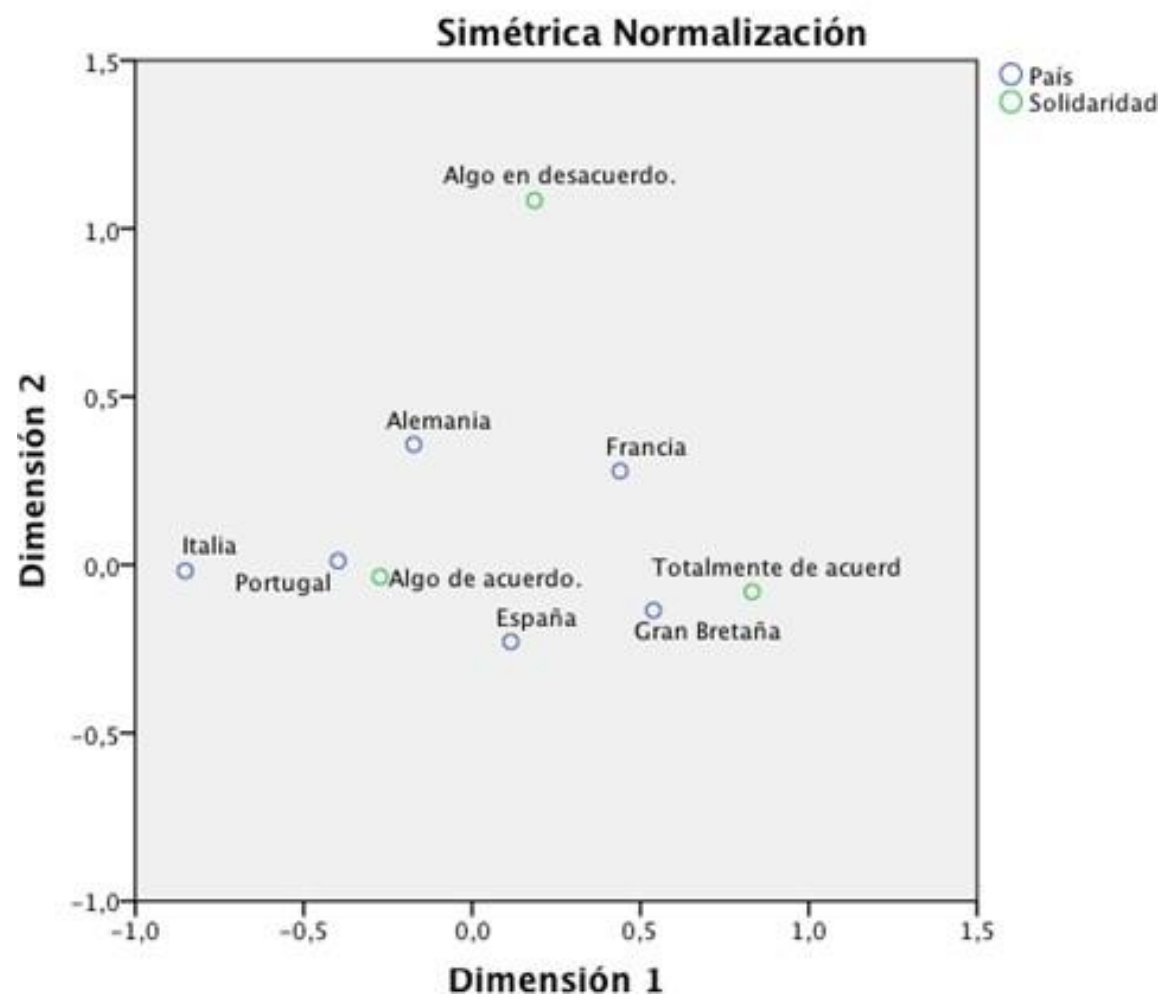




\subsection{Ciudadanía.}

El valor de la ciudadanía es contrario al dejar hacer a los demás, al pasotismo con que algunas veces se juzga a la juventud actual. La ciudadanía significa participación y colaboración, especialmente en la vida pública. "La ciudadanía se optimiza con la participación, con la colaboración, con el esfuerzo encaminado a mejorar el bienestar de la comunidad" (Monreal, Mateos, et alter. 2012, 107-8). En nuestro estudio se ha planteado una cuestión relacionada con la participación de la institución europea.

El $70,2 \%$ de los grupos de jóvenes se manifiestan a favor de que cualquier persona pueda ser elegida para el Parlamento Europeo. En el análisis de correspondencias dos dimensiones explican el $93,4 \%$ de la inercia. Una asociada con las categorías de "totalmente de acuerdo", "algo de acuerdo" y "algo en desacuerdo" $(85,7 \%)$ y la otra $(7,7 \%)$ asociada a "totalmente en desacuerdo".

El análisis del gráfico resulta esclarecedor en este apartado ya que la dimensión se encuentra con contribuciones de columna formadas por categoría a favor y en contra, así que observando el gráfico 7, Gran Bretaña y Portugal se asocian con la categoría "algo de acuerdo" y cercanos al "totalmente de acuerdo", Italia con algo de acuerdo". Alemania sería la más asociada con "algo en desacuerdo". Por lo tanto, británicos y portugueses tienen una percepción más favorable del valor de ciudadanía y menos los grupos de jóvenes alemanes.

\section{Gráfico 7. Puntos de columna y de fila}

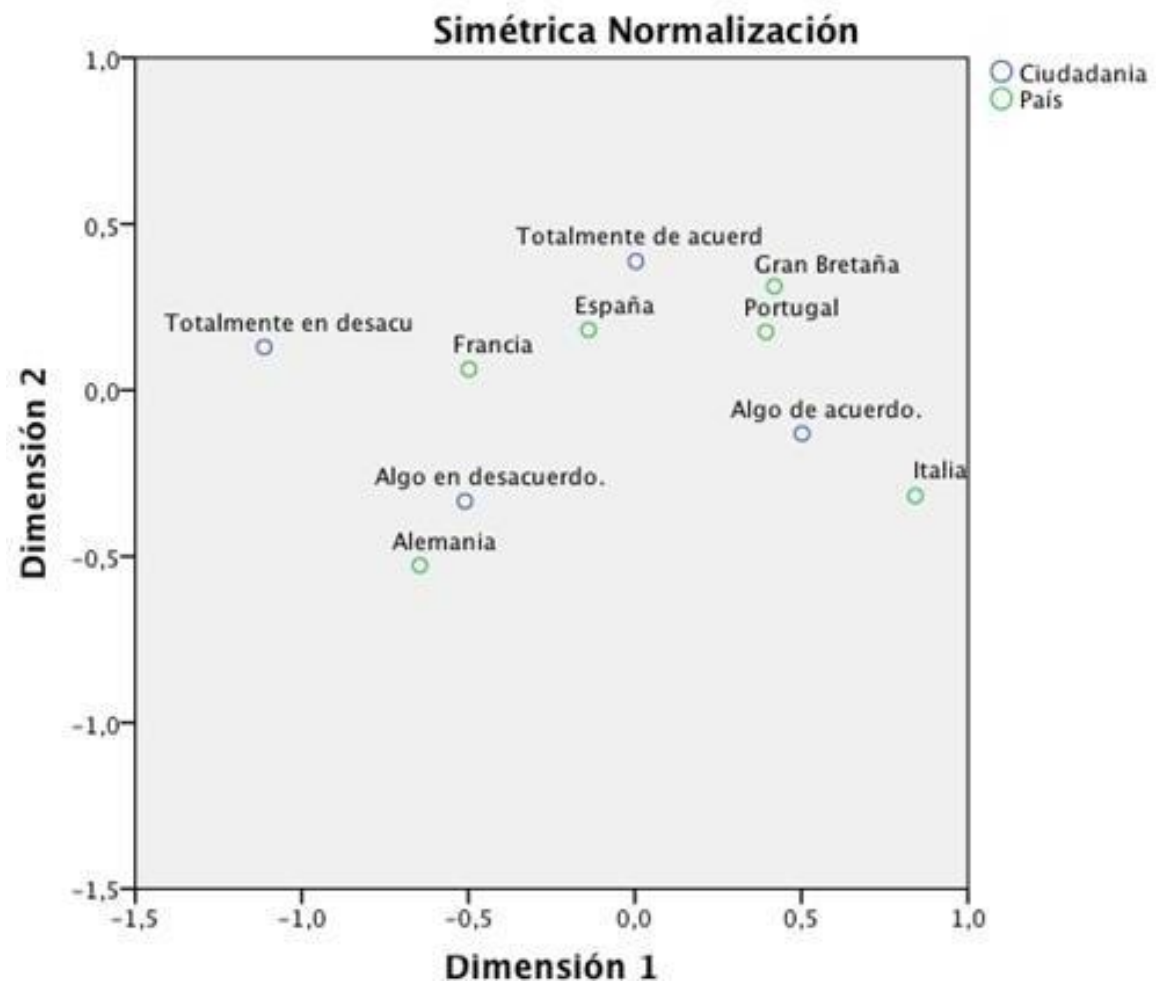




\subsection{Justicia.}

El valor de la justicia permite conocer, respetar y hacer valer el derecho de las personas. Dar a cada quien lo que le incumbe. La justicia es necesaria como valor en las relaciones humanas, como principio tiene como fin solucionar los problemas que surgen de la convivencia humana. Este aspecto es el que se ha buscado en las cuestiones planteadas en el estudio, la igualdad ante la ley, la confianza en ella, la no discriminación en su aplicación y la asistencia jurídica a los que no tienen recursos.

El 84,5\% de los grupos de jóvenes analizados se manifiestan a favor de esta concepción de la justicia en sus países. Al analizar en profundidad los matices de cada uno de sus países, dos dimensiones explican el $99,9 \%$ de la inercia, la primera más representativa con el 93,6\% de ella, asociada a las categorías de "algo en desacuerdo", "algo de acuerdo" y "totalmente de acuerdo". La segunda con una contribución inapreciable $(6,3 \%)$ se asocia con la categoría de "totalmente en desacuerdo". Las contribuciones son inapreciables exceptuando la italiana $(, 803)$, aunque a pesar de ellos la concentración de todos los países, incluyendo este caso italiano asociado a "algo en desacuerdo" (gráfico 8) no permite perfilar con carácter diferencial, en el valor de la justicia, algún país con las categorías estudiadas.

\section{Gráfico 8. Puntos de columna y de fila}

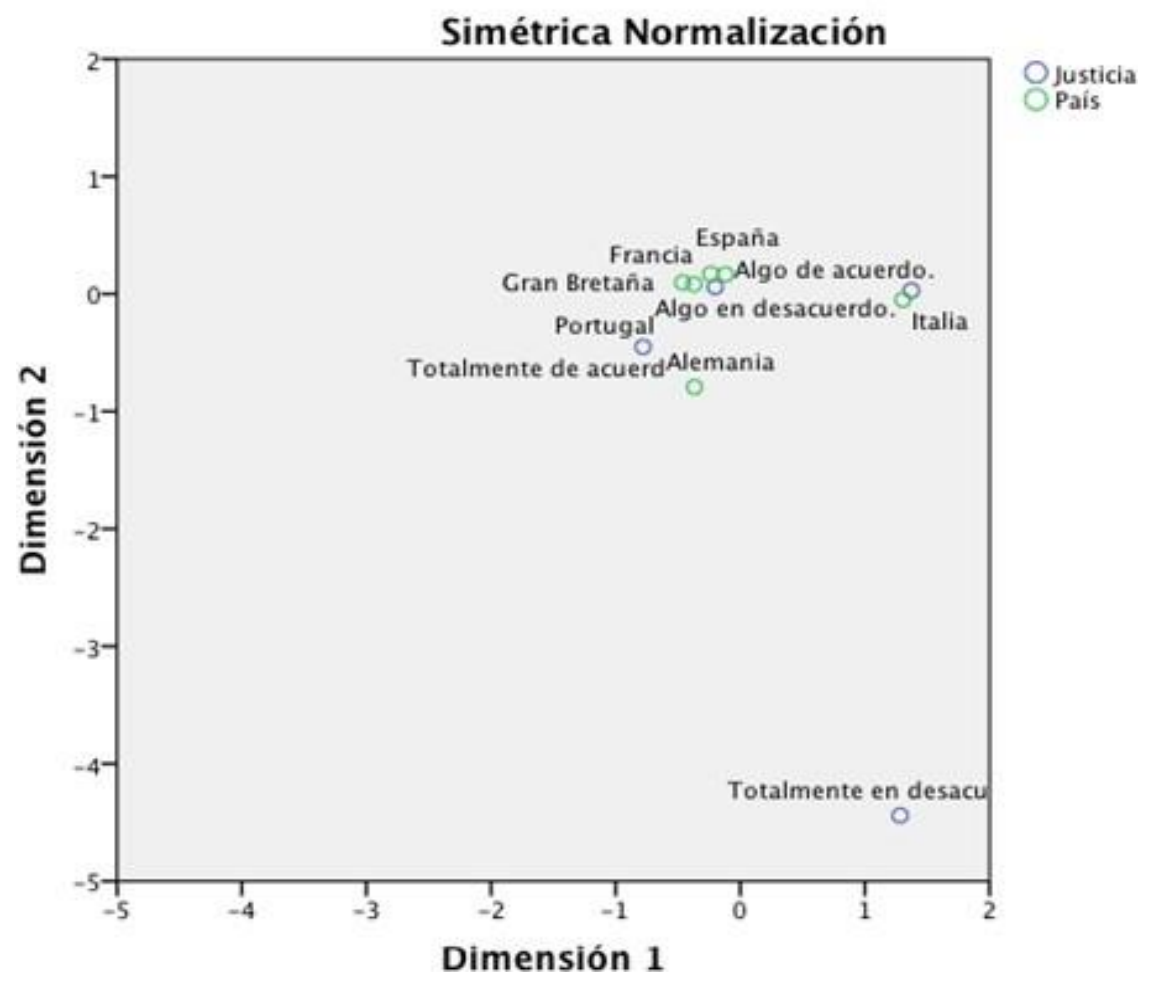




\section{Conclusiones.}

Los jóvenes analizados confirman la idea de que en el momento en el que vivimos y con la ayuda de la televisión, la radio, los soportes digitales por los videos musicales, los juegos de ordenador, el cine y las redes sociales convergen en estilos de vivir y en valores de un mundo globalizado. Las industrias dedicadas al mundo del joven, realizan modelos que se difunden por igual en todo el planeta, de tal manera que no resulta nada complicado reconocer en países distintos los grupos juveniles pertenecientes a tendencias comunes que trascienden lo nacional. Sin embargo, la juventud, dotada siempre del espíritu de libertad sobre sí misma, a captar aquellas tendencias a sus propias cultura locales y regionales que harán coexistir el homo gene ida y la diversidad e incluso, como rasgo de identidad, a valorar lo tradicional como cualidad de afianzamiento frente a la globalización.

Es innegable la influencia de la cultura conviviendo con las nuevas tendencias universalistas. Aun así, la juventud actual, la "generación de la red" (Don Tapscott, 1998) que vive bajo el impacto cultural de las nuevas tecnologías mantiene visiones de la vida y del mundo apegadas también a los contextos culturales de procedencia, los resultados de este estudio confirman estas apreciaciones.

En el análisis hemos podido comprobar como las opiniones de los jóvenes difieren en las categorías de apreciación con las que han manifestado su percepción sobre cada uno de los valores estudiados, permitiendo que pueda realizarse un acercamiento definitorio a las características singulares de cada grupo o país a través de los valores constitucionales.

Los grupos españoles se distinguen por su acercamiento, apreciación, específica a los valores de la dignidad humana, el respeto a la vida y la integridad de las personas y de manera general a la igualdad y la justicia.

Para los franceses los valores específicos o la vida, la integridad de la persona y la solidaridad, y en general la igualdad y la justicia.

En el caso de los británicos específicamente se acercan a los valores de la vida, la solidaridad y la ciudadanía y genéricamente a los valores de la cuarta y la justicia. Para los jóvenes de Alemania la igualdad y la justicia. También para los italianos. Finalmente, para los portugueses los valores de la libertad y la ciudadanía en concreto y la libertad y la justicia en general.

Todos comparten la preocupación porque en la sociedad tempere le guardad si la justicia. Así también... Confirma el pensamiento de los jóvenes en cuanto a que todos deben disfrutar los mismos derechos y libertades, aunque para ello deban observar con su conducta las leyes o los derechos humanos fundamentales.

La solidaridad, destaca en la juventud francesa, no representa los jóvenes actuales menos solidarios más individualistas o como se constata en autores clásicos de la sociología juvenil (Oriel, 1991; Elzo, 1990; Gervilla, 2000; etc.) Un relativismo y ausencia de compromisos sociales como si hubieran desplazado esta responsabilidad a la propia sociedad. 
El respeto por la vida, la integridad I comida humana, que perfilar jóvenes españoles, sigue la estela de los resultados de estudios (Pérez Serrano y otros, 2003) que hablan de una juventud que considera como sociedad ideal aquella en la que predomina en la paz, la igualdad entre géneros, la oposición el racismo, las libertades individuales y el rechazo a la marginación.

Finalmente, el valor de la ciudadanía interesada por los británicos y portugueses, resulta singularmente preocupante porque los jóvenes, nos han entusiastas de la participación social (Pérez Serrano, 2003) desconfiando de los poderes públicos (Musitu,Monreal, Mateos, 2011), o se manifiestan alejados de los valores de la sociedad político-cultural a la que pertenecen.

Estas particularidades y contradicciones en muchos casos, permiten una reflexión sobre la educación en valores, coincidiendo con Mayor Zaragoza $(2009$, 42) cuando afirma que hay que llenar "de educación en valores tantos espacios ocupados hoy por los indeseables huéspedes de la confusión, la violencia, el sometimiento, la indiferencia; la educación como un luminoso e iluminado camino hacia la paz, la emancipación de los seres humanos, el desprendimiento y la solidaridad". Y con Delors $(1996,98)$ al destacar en la educación de los jóvenes: "La promoción de la ciudadanía activa y la cohesión social"

Es necesaria una educación para los valores constitucionales o, dicho de otro modo, una educación para la Ciudadanía en la que también la Universidad debe sumarse puesto que la Universidad se vincula a los intereses sociales, a la participación para profundizar la democracia y para el desarrollo, la búsqueda y la consecución de una sociedad más justa.

\section{Bibliografía.}

- Agudelo, A., Cava, M. J. y Musitu, G. (2001): Un análisis intercultural de la socialización familiar y los valores en adolescentes. Escritos de Psicología, 5, 70-80.

- Amador Muñoz, L. (2005). Cambio Social y Voluntariado. El Papel de la Comunidad Universitaria., en $8^{\circ}$ Congreso Estatal de Voluntariado. Granada. Juan de Andalucía. Consejería de la Presidencia

- Anduiza Perea, E. (2001). Actitudes valores y comportamiento político de los jóvenes españoles y europeos: un estudio comparado. Madrid. INJUVE.

- Antón, A. (200). Jóvenes y sindicatos. Madrid: GPS- Fundación sindical de Estudios.

- Barton (1962) Measuring the values of inddividueals. Religious Education Research Supplement, 57(4), 62-97.

- Berlín, I. (2000). Cuatro ensayos sobre la Libertad. Madrid: Alianza Editorial.

- Bernete García, F. (1995). "Evolución de los indicadores sobre valores políticos de la juventud, utilizados en encuestas nacionales". Revista de La Facultad de Ciencias de la Información,11, 93-107.

- Bernete García, F. (1998). Informe "Juventud en Asturias" 1996. Oviedo. Principado de Asturias, Consejería de Cultura.

- B.O.E de 29 de diciembre de 2006, Real Decreto 1631.

- Castro, A. y Nader, M. (2006): La evaluación de los valores humanos con el Portrait Values Questionnaire de Schwartz. Interdisciplinaria, 23 (2), 155-174. 
- Cortina. A. (1998). Los ciudadanos como protagonistas, Barcelona, Galaxia Gutenberg/Círculo de Lectores,

- Cortina. A. (2000). El mundo de los valores. Ética mínima y Educación. Bogotá. El Búho.

- Delors, J. (1996): "Los cuatro pilares de la educación" en La educación encierra un tesoro. Informe a la UNESCO de la Comisión internacional sobre la educación para el siglo XXI. Madrid, España: Santillana/UNESCO (pp.91-103)

- Durkheim, E. (1975): Educación y sociología. Barcelona, Península.

- González L.J., y Marquinez, G. (2004). Valores éticos para la convivencia. Bogotá. El Búho.

- Greer, P. R. y Ryan, K. (1989): How to answer the hard question about moral education. The American School Journal, 176, 26-28.

- León, L. (2005). Comunicación presentada en las II Jornadas Internacionales sobre políticas educativas para la sociedad del conocimiento. Granada. Marzo 2007

- Martínez, M. (2004). ¿Qué significa educar en valores hoy? Barcelona: Octaedro.

- Mateos, F. (1998): Los valores sociales en los jóvenes del Estrecho de Gibraltar: En J. Lara; R. Marín y G. Pérez (Coord.). Educación Multicultural para la Paz. Sevilla. Algaida.

- Monreal, M. C. (2009). Valores y actitudes de la juventud europea ante las instituciones democráticas. Reflexiones en la Juventud en el S. XXI. Revista de Estudios de Juventud del S. XXI, 87, pp. 81-94.

- Orizo, A. (1991). Los nuevos valores de los españoles. Madrid: Fundación Santa María.

- Orizo F.A. Y Elzo, J. (Drs., 2000). España 2000: entre el localismo y la globalidad. La encuesta europea de valores en su tercera aplicación: 1981-1999. Madrid. Universidad de Deusto/Ediciones S.M.

- Ortega y Gasset, J. (1983): Obras completas. Madrid, Alianza editorial.

- Pérez Serrano, G (1997): Cómo educar para la democracia. Madrid. Editorial Popular.

- Pérez Serrano, G.; Mateos Claros, F.; López Noguero, F., y Otros (2003): Valores y actitudes democráticas de los Jóvenes Andaluces. Sevilla. IAJ: Consejería de la Presidencia.

- Prieto, L. B. (1984). Principios generales de la educación. Caracas: Monte Ávila. P 186.

- Rokeach, M. (1968). Beliefs, attitudes and values. San Francisco: Josse-Bass.

- Rokeach, M. (1979). The nature of human values. New York: Free Press.

- Schwartz, S. H. (1994). Are these universal aspects in the structure and content of human values? Journal of Social Issues, 50, 19-45.

- Thiebaut, C. (1998-2000) Conceptos fundamentales de Filosofía. Madrid, España. Alianza Editorial, S.A.

- Tapscott, D. (1998). Growing Up Digital: The Rise of the Net Generation, New York, McGraw-Hill. 